\title{
SUBADDITIVE STOCHASTIC PROCESSES
}

\author{
BY MICHAEL D. SMELTZER
}

\section{Communicated by Alexandra Bellow, February 18, 1977}

Subadditive stochastic processes were first given a mathematical formulation by J. M. Hammersley and D. J. A. Welsh in 1965 in a paper on first passage percolation problems [8]. They developed a convergence theory for these processes, but their results were rather incomplete. In [10] J. F. C. Kingman modified the definition slightly as follows: Let $(\Omega, \mathrm{B}, P)$ be a probability space. $Y_{m, n}(\omega), m<n$ nonnegative integers, is called a stationary subadditive process with finite time constant if there exists a measure-preserving transformation $T$ which maps $\Omega$ onto $\Omega$ such that

(i) $Y_{m+1, n+1}(\omega)=Y_{m, n}(T \omega)$ for all $\omega \in \Omega, m<n$,

(ii) $Y_{0, m+n}(\omega) \leqslant Y_{0, m}(\omega)+Y_{0, n}\left(T^{m} \omega\right)$ for all $\omega \in \Omega, m, n \geqslant 1$,

(iii) $Y_{0, n}(\omega) \in L^{1}(\Omega, \mathbf{B}, P)$ and $\mathbf{E}\left(Y_{0, n}(\omega)\right) \geqslant-A n$ for some positive constant $A, n \geqslant 1$.

The time constant $\gamma$ is defined by

$$
\gamma=\lim _{n \rightarrow \infty} \frac{\mathbf{E}\left(Y_{0, n}(\omega)\right)}{n}=\inf _{n \geqslant 1} \frac{\mathbf{E}\left(Y_{0, n}(\omega)\right)}{n}-\infty<\gamma<\infty .
$$

In making this definition, Kingman noted that all of the examples considered by Hammersley and Welsh, in fact, satisfy this modified form. Of course, one can construct examples of subadditive processes in the sense of Hammersley and Welsh which are not subadditive processes in the sense of Kingman, but the examples tend to be contrived.

Using this definition, Professor Kingman was able to obtain an almost everywhere convergence theorem corresponding to Birkhoff's ergodic theorem; $Y_{0, n}(\omega) / n$ converges a.e. Kingman's proof is interesting because it yields a decomposition of a subadditive process with time constant $\gamma$ as the sum of a stationary additive process and a nonnegative, stationary, subadditive process with time constant 0 . However, the proof is very complicated, using various weak compactness arguments and sophisticated results of Yosida and Hewitt on purely finitely additive set functions. Burkholder was able to simplify the argument some [11], but he still needed the very esoteric theorem of Komlós.

This paper gives a more direct proof utilizing upcrossing techniques yielding an upcrossing inequality similar to that of J. Doob for martingales. The

AMS (MOS) subject classifications (1970). Primary 60F15; Secondary 28A65. 
method of argument is due to E. Bishop. The idea is to count the number of upcrossings and then downcrossings of two planes in 3-space by a process which is closely related to the process $Y_{0, n}(\omega) / n$. The counting procedure is intimately related to the stationarity of the process. And, in the space with coordinates given by $(u, v, z)$, the planes are given by $z=v b-u a$ and $z=v a-u b, a<b$ $\in \mathbf{R}$.

THEOREM. Let $(\Omega, \mathbf{B}, P)$ be a probability space. Let $Y_{m, n}(\omega)$ be a stationary subadditive stochastic process with finite time constant. Let $a<b \in \mathbf{R}$. Define

$$
\begin{gathered}
W_{N}(\omega)=\max \left\{n \mid 0 \leqslant u_{1}<v_{1}<, \ldots,<u_{n}<v_{n} \leqslant N,\right. \\
v_{i} b-u_{i} a \leqslant Y_{u_{i}, v_{i}}(\omega) \quad 1 \leqslant i \leqslant n, \quad \text { and } \\
\left.Y_{v_{i}, u_{i+1}}(\omega) \leqslant u_{i+1} a-v_{i} b \quad 1 \leqslant i \leqslant n-1\right\} .
\end{gathered}
$$

Under these assumptions,

$$
\int_{\Omega} W_{N}(\omega) d P \leqslant \frac{1}{b-a} \int_{\Omega}\left(Y_{0,1}(\omega)-a\right)^{+} d P .
$$

Using this theorem, it is not too dificult to prove the result of Kingman:

Corollary. If $(\Omega, \mathbf{B}, P)$ is a probability space, and $Y_{m, n}(\omega)$ is a stationary subadditive process with finite time constant $\gamma$, then there exists a random variable $\widetilde{Y}(\omega)$ such that $Y_{0, n}(\omega) / n$ converges almost everywhere to $\widetilde{Y}(\omega)$, and $\mathbf{E}(\widetilde{Y}(\omega))=\gamma$.

The method of proof for the corollary consists of showing that

$$
P\left\{\omega \mid \liminf _{n \rightarrow \infty} Y_{0, n}(\omega) / n<a<b<\limsup _{n \rightarrow \infty} Y_{0, n}(\omega) / n\right\}=0 .
$$

Other than the theorem, the only property used is that if $P(B)>0$ for some set $B$, then up to a set of measure zero, $\bigcup_{k=j}^{\infty} T^{k}(B)=\bigcup_{k \in Z} T^{k}(B)$.

\section{REFERENCES}

1. G. D. Birkhoff, Proof of the ergodic theorem, Proc. Nat. Acad. Sci. 17 (1931), 656-660.

2. E. Bishop, An upcrossing inequality with applications, Michigan Math. J. 13 (1966), 1-13. MR 33 \#2772. $36 \# 4930$.

3. - Foundations of constructive analysis, McGraw-Hill, New York, 1967. MR

4. L. Breiman, Probability, Addison-Wesley, Reading, Mass., 1968. MR 37 \#841.

5. J. L. Doob, Stochastic processes, Wiley, New York, 1953. MR 15, 445.

6. A. Garsia, A simple proof of $E$. Hopf's maximal ergodic theorem, J. Math. Mech. 14 (1965), 381-382. MR 35 \#338.

7. J. M. Hammersley, Postulates for subadditive processes, Ann. Probability 2 (1974), 652-680. MR 51 \#6947.

8. J. M. Hammersley and D. J. A. Welsh, First-passage percolation, subadditive processes, stochastic networks, and generalized renewal theory, Bernoulli-Bayes-Laplace Anni- 
versary Volume, edited by J. Neyman and L. M. LeCam, Springer-Verlag, Berlin and New York, 1965. MR 33 \#6731.

9. K. Yosida and S. Kakutani, Birkhoff's ergodic theorem and the maximal ergodic theorem, Proc. Imp. Acad., Tokyo 15 (1939), 165-168. MR 1, 59.

10. J. F. C. Kingman, The ergodic theory of subadditive stochastic processes, J. Roy. Statist. Soc. Ser. B 30 (1968), 499-510. MR 40 \#8114.

11. - Subadditive ergodic theory, Ann. Probability 1 (1973), 883-909. MR $50 \# 8663$.

DEPARTMENT OF MATHEMATICS, YALE UNIVERSITY, NEW HAVEN, CONNECTICUT 06520 